\title{
Cisternostomy for traumatic brain injury-rigorous evaluation is necessary
}

\author{
Franco Servadei ${ }^{1} \cdot$ Angelos Kolias $^{2,3} \cdot$ Ramez Kirollos $^{4} \cdot$ Tariq Khan $^{5} \cdot$ Peter Hutchinson ${ }^{2,3}$ (ID
}

Received: 9 January 2020 / Accepted: 10 January 2020 / Published online: 21 January 2020

(C) Springer-Verlag GmbH Austria, part of Springer Nature 2020

Traumatic brain injury (TBI) remains a major public health problem globally [14]. Indications for neurosurgical intervention following TBI can be broadly categorised as (1) evacuation of haematoma, (2) control of intracranial pressure (with or without haematoma evacuation), (3) elevation of depressed skull fractures, (4) repair of skull base fractures with or without CSF fistula, (5) treatment of hydrocephalus, and (6) cranial reconstruction [17].

Cisternal opening is a well-embedded microsurgical technique in neurosurgical practice for vascular and skull base pathologies. Its application in the context of TBI, in combination with insertion of an external cisternal drain which stays in place for a few days post-operatively, has been termed cisternostomy. The first publication of cisternostomy dates back to 2013 [2]. That paper reported the rationale around cisternal opening in TBI and also presented the results of a single-surgeon series of patients treated with decompressive craniectomy (DC) plus cisternostomy (272 patients) and cisternostomy alone (476 patients). Details about the research protocol are sparse but the authors reported that the mean GOS at 6 weeks was 3.7 and 3.9 respectively. The lack of details about (1) the research protocol, (2) the clinical TBI

This article is part of the Topical Collection on Brain trauma

Peter Hutchinson

pjah2@cam.ac.uk

1 Department of Neurosurgery, Humanitas University and Research Hospital, Milan, Italy

2 Division of Neurosurgery, Department of Clinical Neurosciences, Addenbrooke's Hospital and University of Cambridge, Cambridge Biomedical Campus, Box 167, Cambridge CB2 0QQ, UK

3 NIHR Global Health Research Group on Neurotrauma, University of Cambridge, Cambridge, UK

4 National Neuroscience Institute and Duke-NUS Medical School, Singapore, Singapore

5 Department of Neurosurgery, Northwest General Hospital and Research Center, Peshawar, Pakistan algorithm followed, and (3) the occurrence of complications mean that no firm conclusions can be drawn on the basis of that paper.

Since then, we identified another 13 papers (PubMed search with the term "cisternostomy" or "cisternotomy" in the title). Only 2 of these 13 papers include patient data and both were single-patient case reports $[6,15]$. The majority of the remaining papers are narrative reviews or letters and have mostly discussed the proposed physiological rationale of this operation, namely, reversal of cerebrospinal fluid shift oedema of the brain [3]. At this juncture, it has to be stated that the ability of this operation to reverse this type of oedema is an entirely theoretical concept with no direct evidence proving this hypothesis.

The accompanying paper by Giammattei et al. presents the results of 40 patients with severe TBI (sTBI) treated at Centre Hospitalier Universitaire Vaudois in Lausanne between 2013 and 2018. This was a retrospective study. Of the 40 patients, 22 had a decompressive craniectomy (DC), while 18 had a DC with a cisternostomy. The decision as to whether a cisternostomy would be performed was only dependent on the availability of a neurosurgeon with vascular surgery expertise, rather than any clinical characteristics. This raises questions around the statement that "waiver of consent was granted because the procedure was part of our written algorithm for the management of sTBI", given that the treatment algorithm would be different depending on who was the on-call neurosurgeon. This issue along with the fact that the published data on cisternostomy for TBI are very sparse indicates that the study should have happened prospectively in the context of a research protocol approved by an ethics committee [8].

The very limited sample size and the retrospective nature are major sources of bias. With the "subgroup analysis" (primary vs secondary procedure), the sample size becomes even more limited. On this basis, all outcome data should be treated cautiously and, at best, as evidence that in the hands of experienced vascular neurosurgeons the addition of cisternostomy to DC does not seem to lead to worse outcomes. 
Another concern is that these findings cannot be necessarily translated to a typical neurosurgical trauma service. Firstly, polytrauma patients were excluded. About one-third of sTBI patients have major extracranial injuries and these are independently associated with mortality [20]. Secondly, the workload of the trauma service at Centre Hospitalier Universitaire Vaudois seems to be very limited, with only 50 operations for sTBI over 6 years - which is less than one per month. Di Cristofori et al. have previously raised concerns as to whether a typical neurosurgical service would be able to offer cisternostomy routinely, assuming that its effectiveness is proven [5].

Although in the current paper, cisternostomy was offered as an adjunct to DC, the same group from Lausanne have published a case report of stand-alone cisternostomy (i.e., without DC) and have postulated that cisternostomy could become a stand-alone treatment in the future, assuming that multi-centre clinical studies prove its effectiveness [6]. At this juncture, it is useful to summarise the evidence behind DC.

Decompressive craniectomy is an operation which involves removal of a large cranial bone flap [12]. It can be used as a primary procedure - undertaken at the same time as evacuation of an intracranial haematoma - or secondary with the aim of alleviating elevated intracranial pressure (ICP) refractory to medical management. Several case series and cohort studies have been published but nowadays there is also evidence from randomised trials. Evidence from the 5 published randomised trials of DC for TBI has been recently summarised as follows [13]:

1) unilateral or bifrontal $\mathrm{DC}$ used as a last-tier therapy for patients with severe, sustained, and refractory posttraumatic intracranial hypertension leads to a substantial mortality reduction but increases disability (both lower (dependent) and upper (independent at home) severe disability) compared with medical management [9]

2) early neuroprotective bifrontal DC for mild to moderate intracranial hypertension is not superior to medical management for patients with diffuse TBI [4]

3) a large fronto-temporo-parietal DC (around $15 \mathrm{~cm}$ ) is superior to a small unilateral DC (around $8 \mathrm{~cm}$ ) for patients with unilateral hemispheric swelling with large or smallsize contusions $[10,18]$

4) a small pilot study found a trend towards improved survival and functional outcomes with bitemporal decompression compared with medical management in children with post-traumatic intracranial hypertension [19].

Comparing the evidence for DC with that for cisternostomy (refer to the third paragraph), one can easily see that the latter still has a long way to go before it can be considered a "standard treatment" for TBI.

Despite the fact that cisternal opening is well embedded in neurosurgical practice for vascular and skull base pathologies, cisternostomy can be considered a novel surgical approach for TBI. This raises a number of important issues. Neurosurgery has been intimately related to innovation since its origins. Innovations, such as the operating microscope, CT and MR imaging, and endoscopy and image guidance, just to name a few, have brought our specialty at the forefront of technological advances but most importantly have been readily translated to improved outcomes, including safety, for our patients. Nowadays, technological innovations promise less dramatic improvements than the introduction of the microscope or CT scanning for example. Nonetheless, small incremental benefits can still be important and make a significant contribution in our efforts for making neurosurgery safer with preservation of quality of life for our patients. On the other hand, a number of innovations that often appear appealing on paper have no added benefits when subjected to rigorous evaluation. In fact, this phenomenon, due to its relatively frequent occurrence, has been termed "medical reversal" $[7,16]$. Medical reversals are "practices that have been found, through randomised controlled trials, to be no better than a prior or lesser standard of care" [7].

The literature abounds with such examples. In the field of cardiology, revascularisation via percutaneous coronary intervention (PCI) for patients with chronic stable angina was widely practised but was not found better than a low-cost regimen of medical therapy and lifestyle modification when subjected to rigorous evaluation with a randomised trial [1]. In the field of orthopaedics and musculoskeletal medicine, autologous platelet-rich plasma has been used extensively in recent years for a variety of musculoskeletal injuries. However, a recent large randomised trial found no evidence to indicate that injections of platelet-rich plasma can improve objective muscle tendon function, patient reported function, or quality of life after acute Achilles tendon rupture compared with placebo [11]. The authors of this trial rightly concluded that "a sound theoretical mechanism and clinical indication are not enough" [11].

As doctors, we have a duty of care to our patients. In our view, this also extends to the rigorous evaluation of novel techniques, approaches, and devices [8]. This is important from the non-maleficence and safety perspective. Moreover, conducting high-quality studies is essential in order to ensure that novel techniques can be rapidly incorporated in clinical recommendations and therefore maximise patient benefit, if found to be effective. Cisternostomy for sTBI should be treated as a novel technique but there is a lack of high-quality studies to support its use outside the context of research. This is in contrast to DC, with its use supported by evidence from randomised trials, as mentioned above. The responsibility lies with the proponents of cisternostomy to conduct prospective, randomised, controlled studies that will clarify whether it is effective or not. 


\section{Compliance with ethical standards}

Conflict of interest Franco Servadei has received personal fees from Takeda Pharmaceutical Company, grants and personal fees from Integra LifeSciences, and grants and personal fees from Finceramica SpA. The rest of the authors declare that they have no conflict of interest related to this manuscript.

\section{References}

1. Boden WE, O'Rourke RA, Teo KK et al (2007) Optimal medical therapy with or without PCI for stable coronary disease. N Engl J Med 356(15):1503-1516

2. Cherian I, Yi G, Munakomi S (2013) Cisternostomy: replacing the age old decompressive hemicraniectomy? Asian J Neurosurg 8(3): 132-138

3. Cherian I, Burhan H, Dashevskiy G, Motta SJH, Parthiban J, Wang Y, Tong H, Torregrossa F, Grasso G (2019) Cisternostomy: a timely intervention in moderate to severe traumatic brain injuries: rationale, indications, and prospects. World Neurosurg 131:385-390

4. Cooper DJ, Rosenfeld JV, Murray L et al (2011) Decompressive craniectomy in diffuse traumatic brain injury. N Engl J Med 364(16):1493-1502

5. Di Cristofori A, Gerosa A, Panzarasa G (2018) Is neurosurgery ready for cisternostomy in traumatic brain injuries? World Neurosurg 111:427

6. Giammattei L, Messerer M, Oddo M, Borsotti F, Levivier M, Daniel RT (2018) Cisternostomy for refractory posttraumatic intracranial hypertension. World Neurosurg 109:460-463

7. Herrera-Perez D, Haslam A, Crain T, Gill J, Livingston C, Kaestner V, Hayes M, Morgan D, Cifu AS, Prasad V (2019) A comprehensive review of randomized clinical trials in three medical journals reveals 396 medical reversals. Elife. https://doi.org/10.7554/eLife. 45183

8. Hirst A, Philippou Y, Blazeby J et al (2019) No surgical innovation without evaluation. Ann Surg 269(2):211-220

9. Hutchinson PJ, Kolias AG, Timofeev IS et al (2016) Trial of decompressive craniectomy for traumatic intracranial hypertension. $\mathrm{N}$ Engl J Med 375(12):1119-1130
10. Jiang J-Y, Xu W, Li W-P, Xu W-H, Zhang J, Bao Y-H, Ying Y-H, Luo Q-Z (2005) Efficacy of standard trauma craniectomy for refractory intracranial hypertension with severe traumatic brain injury: a multicenter, prospective, randomized controlled study. J Neurotrauma 22(6):623-628

11. Keene DJ, Alsousou J, Harrison P et al (2019) Platelet rich plasma injection for acute Achilles tendon rupture: PATH-2 randomised, placebo controlled, superiority trial. BMJ. https://doi.org/10.1136/ BMJ.L6132

12. Kolias AG, Viaroli E, Rubiano AM et al (2018) The current status of decompressive craniectomy in traumatic brain injury. Curr Trauma Rep 4 (4):326-332

13. Kolias AG, Paschalis A, Fountas KN, Hutchinson PJ (2019) The value of decompressive craniectomy in traumatic brain injury. Evid. Neurosurg. Springer International Publishing, Cham, pp 5-18

14. Kolias AG, Rubiano AM, Figaji A, Servadei F, Hutchinson PJ (2019) Traumatic brain injury: global collaboration for a global challenge. Lancet Neurol 18(2):136-137

15. Masoudi MS, Rezaee E, Hakiminejad H, Tavakoli M, Sadeghpoor $\mathrm{T}$ (2016) Cisternostomy for management of intracranial hypertension in severe traumatic brain injury; case report and literature review. Bull Emerg Trauma 4(3):161-164

16. Prasad V, Cifu A, Ioannidis JPA (2012) Reversals of Established Medical Practices. JAMA 307(1):37

17. Pujari R, Hutchinson PJ, Kolias AG (2018) Surgical management of traumatic brain injury. J Neurosurg Sci 62(5):584-592

18. Qiu W, Guo C, Shen H, Chen K, Wen L, Huang H, Ding M, Sun L, Jiang Q, Wang W (2009) Effects of unilateral decompressive craniectomy on patients with unilateral acute post-traumatic brain swelling after severe traumatic brain injury. Crit Care 13(6):R185

19. Taylor A, Butt W, Rosenfeld J, Shann F, Ditchfield M, Lewis E, Klug G, Wallace D, Henning R, Tibballs J (2001) A randomized trial of very early decompressive craniectomy in children with traumatic brain injury and sustained intracranial hypertension. Childs Nerv Syst 17(3):154-162

20. van Leeuwen N, Lingsma HF, Perel P et al (2012) Prognostic value of major extracranial injury in traumatic brain injury: an individual patient data meta-analysis in 39,274 patients. Neurosurgery 70(4): 811-818 discussion 818

Publisher's note Springer Nature remains neutral with regard to jurisdictional claims in published maps and institutional affiliations. 